\title{
Analysis and Development of Efficient DSM Engine for Smart Industrial Based on Big Data and IoT
}

\author{
Rupal Chaudhary \\ Assistant Professor, Department of Computer Science, Sir Chhotu Ram Institute of Engineering and \\ Technology, C. C. S. University, Uttar Pradesh, India
}

\begin{abstract}
Savvy social orders have an expanding interest for quality-arranged administrations and framework in an Industrial Internet of Things (IIoT) worldview. Savvy urbanization faces various difficulties. Among them, made sure about vitality Demand Side Management (DSM) is of specific concern. The IIoT renders the mechanical frameworks to malware, digital assaults, and other security chances. The IIoT with the mixture of Big Data investigation can give effective answers for such difficulties. This paper proposes a made sure about and trusted multi-layered DSM motor for a shrewd social society utilizing IIoT-based Big Data examination. The proposed motor uses an incorporated way to deal with accomplish ideal DSM over a Home Area Network (HAN). To upgrade the security of this motor, a payload-based confirmation conspire is used that depends on a lightweight handshake component. Our proposed strategy uses the lightweight highlights of Constrained Application Protocol (CoAP) to encourage the customers in observing different assets dwelling over the worker in a vitality productive way. Likewise, information streams are handled utilizing Big Data examination with MapReduce equal preparing. The proposed confirmation approach is assessed utilizing NetDuino Plus 2 loads up that yield a lower association overhead, memory utilization, reaction time and a vigorous safeguard against different vindictive assaults.
\end{abstract}

Keywords: Demand Side Management, Home Area Network, Smart Societies, Security, Trust.Industrial Internet of Things

\section{INTRODUCTION}

We are living in a period on this planet when $54 \%$ populace is living in metropolitan zones; with an expectation of an expansion up to $66 \%$ by 2050 [1], [2]. This urbanization will bring various difficulties for the leaders in offering different types of assistance and satisfaction of foundation needs. The Internet of Things (IoT) covers the regular objects of physical world by empowering them to connect with different items [3]. It was anticipated that the associated things will increment $31 \%$ in the year 2017, in contrast with 2016, by arriving at 8.4 billion interconnected gadgets and will cross 20 billion by year 2020 [4]. With the approach of IoT, all the moronic gadgets at homes will have the option to hear, tune in and speak with one another by shaping a Home Area Network (HAN). Besides, this data will be shared over the Internet to make it universal [5]. With this data sharing, the idiotic gadgets will change into savvy gadgets to change the human way of life. IoT is a work organization of home/regular articles, and these items might be outfitted with inescapable knowledge [6].

The basic sensors and gadgets in fundamental industry framework with existing IoT are 
interconnected to shape the Industrial IoT (IIoT). Normally, IIoT abuse licenses organizations and customers into mechanical practices and achieve skyscratching creation by decreasing expense. In the interim, IIoT renders the modern frameworks to malware, digital assaults, and various weaknesses. The fuse of items inside the Internet needs an assortment of correspondence models. This need will probably include various novel and clever ill-disposed models to the IIoT [7]. In IIoT, security provisioning is a lumbering undertaking on the grounds that each article has its own extraordinary highlights. The peculiarity of each article, individual, and framework connected to the Internet should be confirmed. The items and arrangements accessible in the market are missing made sure about highlights and they are powerless against a wide scope of security penetrates.

The colossal number of brilliant gadgets in IIoT condition will create information of high volume, high speed, as well as high assortment data (3V) resources, that require new types of preparing to empower upgraded dynamic, knowledge disclosure and cycle advancement called Big Data. Sharda, et al. [8], [9] included veracity, fluctuation, and incentive to these three Vs. The use of cutting edge expository strategies on huge informational indexes is known as Big Bata examination that comprises of two sections, i.e., the Big Data set and investigation. Progression in IIoT is bringing about a lot of valueable information. With the assistance of Big Data innovations and effective AI calculations, there is an incredible forthcoming of investigative administrations to the metropolitan residents and chiefs [10], [11]. A keen society plans to raise the nature of administration (QoS) and advance these administrations for its inhabitants [12].

\section{RELATED WORK}

Web of Things (IoT) is a merger of various innovations to associate and utilize savvy gadgets that would change the every day life practices. Riggins $F$ and Wamba F. [15] proposed a structure that has its premise on the possibility of development of IoT. As per them, the development will occur from checked things to the organization of things. Sun et al. [16] supports a creative idea of savvy associated networks (condensed as SCC). The idea advanced from the keen urban areas to upgrade the current expectations for everyday comforts and satisfy the future needs in a specialized and creative manner.

The proposed model attempts to accomplish a few goals, for example, charge, uneasiness minimization and amplification of privately created vitality; with certain limitations like electric gadgets (fixed, move capable, and flexible), neighborhood vitality generators, and vitality stockpiling and parity and vitality duties.

\section{PROSPECTIVE OF DEMAND SIDE MANAGEMENT IN SMART SOCIETY}

DMS can be additionally be characterized as keeping up the productive use of the vitality by choosing the high need and appeal gadgets at correct time while staying in the endorsed burden and cost cutoff points and as per the predetermined boundaries or obliges [14], [37], [38]. The DSM representation is portrayed in Figure 1. The boundaries which are mulled over in DSM include: 1. Gadget Load: This is the base burden needed to work a particular gadget. 2. Cost: This is the most basic boundary from buyer perspective and it is the connection of time and request and can be diverse at various season of a specific day however in by and large value taxes from vitality provider are viewed as authority in such manner. 


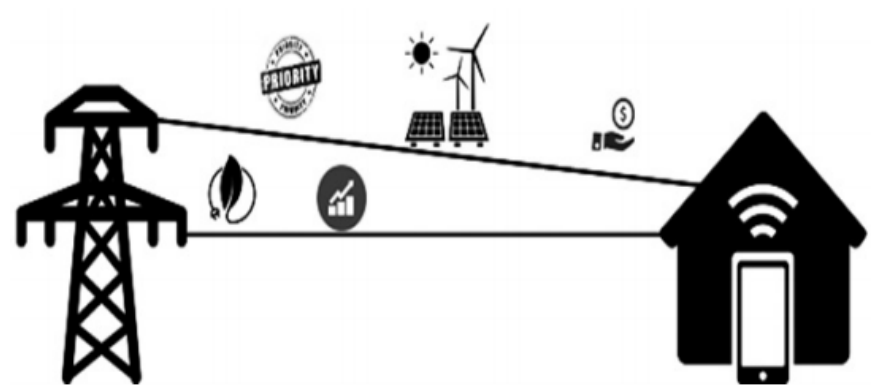

Fig. 1: Demand Side Management: An illustration

3. Burden Limit: It is the most basic boundary from provider perspective; by and large it is a breaking point determined by the provider and it might change as indicated by purchaser hours and levies. 4. Time: The time might be the main factor for huge numbers of the above boundaries as the expense and the heap is estimated by the time unit. 5. Gadget Priority: Priority can be characterized as the proportion of utilization or significance at specific time. Thus, need of a gadget can fluctuate during various hours of a specific day. A particular gadget could be in one and only each need of the accompanying in turn and could change progressively as indicated by circumstance. a. Ongoing: The gadget is completely required respect less of the heap and cost cutoff points or as per the compromises. b. High: The Device is sought after while staying in the heap however not cost cutoff or the other way around yet not both. c. Medium: The gadget is required however when it is achievable to stay in the heap and cost limit. On the off chance that no other compel is determined. d. Low: The gadget is at low need and can be closed down for high need gadget popular. e. Not Required: The gadget will stay off until the need is changed.

From provider forthcoming burden is the essential and from purchaser side its the auxiliary purpose of concern. The fundamental target of DSM is to stay in the heap and cost limits. This can be accomplished with the strategies appeared in Figure 2.
1. Load Shifting: In this technique the load is shifted from peak hours to the off peak hours, in other words it is the way to segregate the devices based on their priority level.

2. Peak Clipping: This is direct load control technique to reduce peak load and achieving the optimum level.

3. Conservation: This technique is used to reduce the energy consumption

4. Load Balancing: This is the technique to balance the load

5. Valley Filling: Valley filling is the opposite of peak clipping. It directly increases the load in offpeak hours. Other possible techniques are shown in the figure 2.

6. Flexible Load: It is a technique that provides the facility of the flexibility the energy.

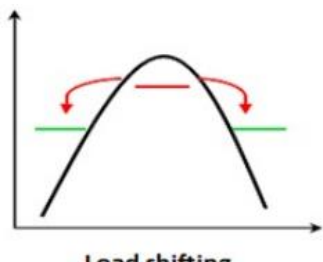

Load shifting

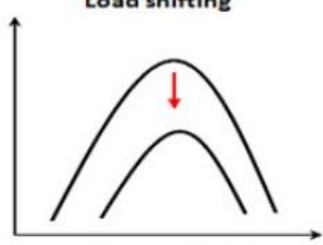

Conservation

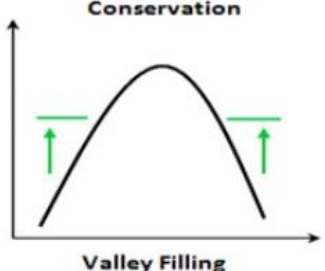

Fig. 2: DSM Techniques

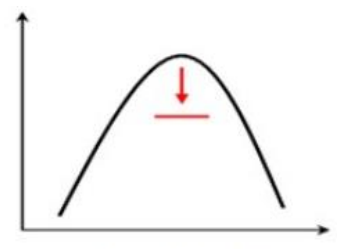

Peak Clipping

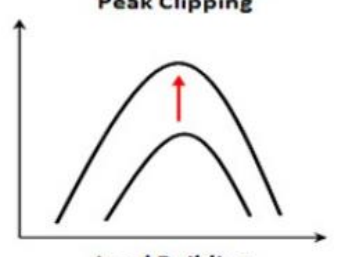

Load Building

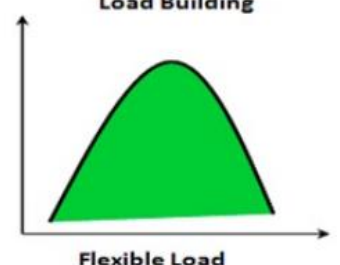

Flexible Load
Trade-offs:

To accomplish the craving results buyer needs to make a few compromises between various wants. Some of them are:

1. Accessibility versus Cost

2. Accessibility versus Burden

3. Cost versus Burden 
Combinatorial enhancement is tied in with finding the ideal set dependent on certain rules given a limited arrangement of items. In the proposed motor, the selection of gadgets depends on the boundaries characterized previously. Likewise, 0/1 Knapsack calculation is utilized in the proposed motor for the choice of gadgets which can be depicted as:

\section{PROPOSED METHODOLOGY}

In this part the exhaustive and detail portrayal of the proposed engineering is talked about. The proposed DMS motor is a multilayered concentrated DMS motor which is made out of 1 . Information/Message Receiving and Pre-Processing, 2. Information Stream Processing, and Decision-Making and User Interface Layers. The situation of the DSM motor in the brilliant social society is appeared in Figure 3 . The HANs appeared in the territory are associated with this motor through LAN or MAN. Before broadly expounding portrayal of each layer of the proposed DSM motor, the review of the engineering is given first.

$$
\text { Max } \sum_{i=1}^{n} \text { DeviceCost }_{i}
$$

subject to

$$
\sum_{i=1}^{n} \text { DeviceLoad }_{i} \leq \text { Loadlimit }
$$

\section{PROPOSED METHODOLOGY}

In this segment the far reaching and detail portrayal of the proposed design is talked about. The proposed DMS motor is a multilayered concentrated DMS motor which is made out of 1 . Information/Message Receiving and Pre-Processing, 2. Information Stream Processing, and Decision-Making and User Interface Layers. The situation of the DSM motor in the shrewd social society is appeared in Figure 3. The HANs appeared in the territory are associated with this motor through LAN or MAN. Before broadly expounding portrayal of each layer of the proposed DSM motor, the review of the engineering is given first.

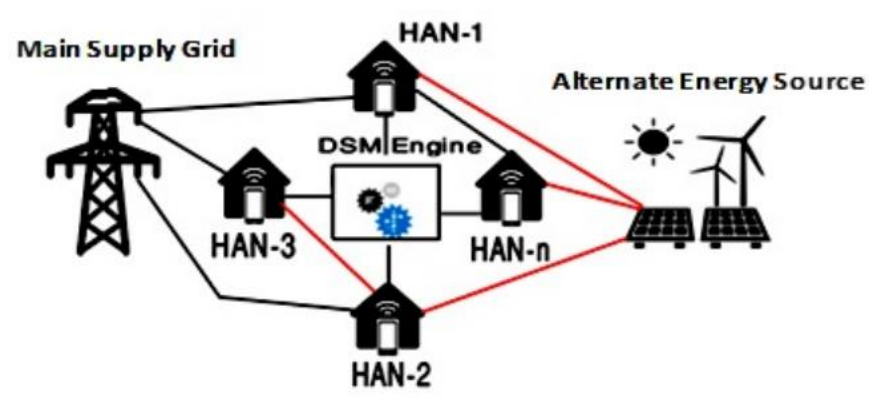

Fig. 3: DSM Engine for Smart Social Society

\section{A. Overview of Proposed DSM Engine}

The review of the multilayered motor is given in this part. An elevated level engineering (review) of the proposed DSM motor is appeared in the Figure 4. The Architecture comprises of different layers including various parts. Most importantly the information created by various HANs associated with the proposed motor are gathered by the message collector. The message distinguishing proof and verification is performed at this stage. The proposed design uses the payload based shared confirmation plot so as to recognize the message and give secure association. The payload confirmation utilizes the CoAP as fundamental convention to meet the prerequisites. The message collector is liable for keys trade and in general confirmation. A while later, this information is sent to the pre-preparing for eliminating the oddities from the crude information as the information got by message recipient is the crude information securing from the external IoT information sources. These irregularities may incorporate the missing qualities, invalid information, untrustworthy information and so on the message recognizable proof cycle is additionally performed at this stage. A short time later, the filtration and extraction is acted so as to channel the information and eliminate the commotion. When the 
preprocessing is done, the information is fit to be handled.

\section{B. Proposed Demand Side Management Engine}

The proposed DMS motor is a multilayered incorporated DMS motor for savvy social orders which is made out of 1. Information/Message Security and Pre-Processing Layer, 2. Information Stream Processing Layer, and Decision-Making and User Interface Layer which are is graphically portrayed in Figure 5. The comprehensive clarification of each layer of the proposed motor is given in the prospective section. 1. Information/Message Security and Pre-handling Layer: This is the primary layer of the proposed DSM motor. This layer is legitimately associated with the IoT information sources (HANs). The information is first gathered and validated at this stage. The information from HANs is gotten as messages which can be one of two kinds: 1. HAN arrangement message, and 2. gadget message. HAN setup message contains the setting/inclinations data of a specific HAN. This kind of message may contain HAN id, Initial needs all things considered, max load breaking point, and max cost limit. Gadget message contains the setting data of a specific gadget containing the data about a specific gadget, for example, gadget id, burden, need, and status. In this manner, most importantly when the information is gathered from information sources, the message recognizable proof cycle is done to give secure and confided in information. Payload based Mutual Authentication Scheme. The payload validation conspire is like CoAP in light of the fact that it utilizes the CoAP as fundamental convention to meet the prerequisites. Nonetheless, the CoAP-based executions for IoT are reliant on DTLS for the secured move of assets among the articles. This situation is portrayed in Figure 5 in the security layer.

For the confirmation, the security highlights into CoAP are included proposed plan to make it more proficient, hearty, and secure against different vindictive exercises. Differentiating the encryption procedures dependent on DTLS, the proposed technique offers validation utilizing the payload of messages traded among the customers and the worker. Both the worker and customer face each other during the cycle of verification. The entire cycle is completed utilizing 4 handshake messages where, the payload of each message is kept a limit of 256 pieces as appeared in Figure 6.

The proposed conspire is practiced utilizing the four stages which are

1. Meeting dispatching,

2. Worker challenge,

3. Customer answer and challenge, and

4. Worker answer.

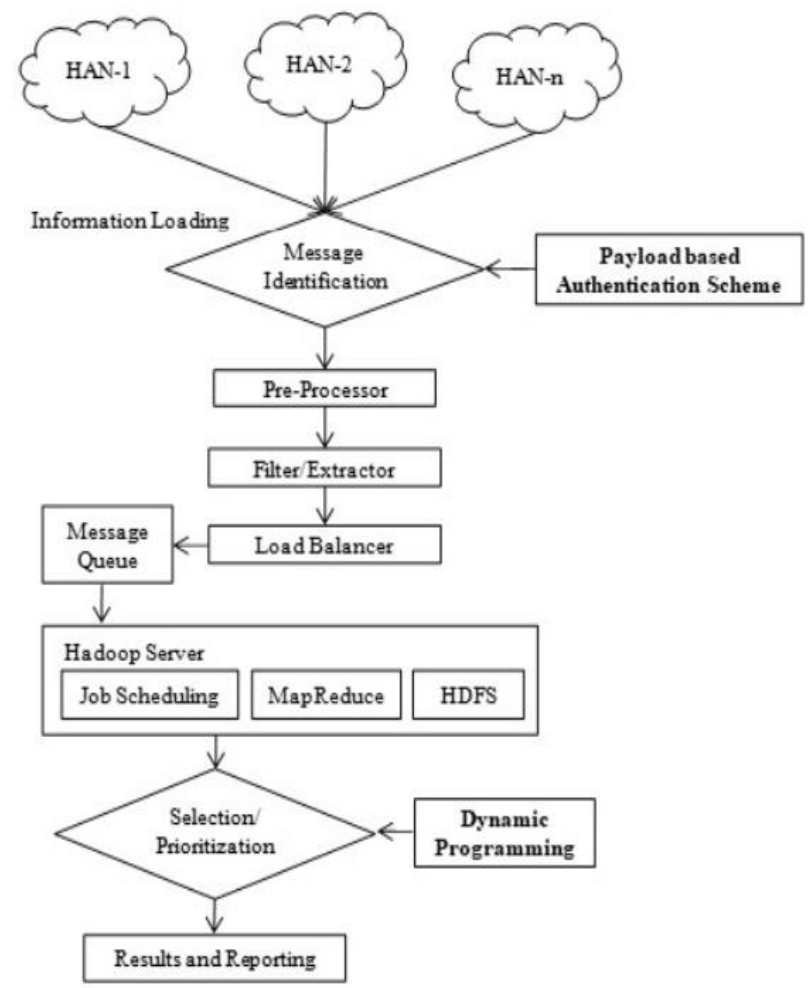

Fig. 4: Overview of DSM Engine

\section{A. Data Reduction,}

Information decrease can be made commonsense to get a diminished portrayal of the datasets that is littler generally in amount, yet intently safeguards the trustworthiness of the first information. Furthermore, 
preparing and examination on the decreased dataset is significantly more proficient yet produce nearly similar scientific outcomes. Information decrease commonly applied on gigantic information that don't present many esteemed information. Moreover, information change is performed. The fundamental motivation behind the change is to restrict the estimation of information to a specific restricted range. We favored the MinMax procedure to change a particular worth $\mathrm{X}$ to another worth $\mathrm{Y}$.

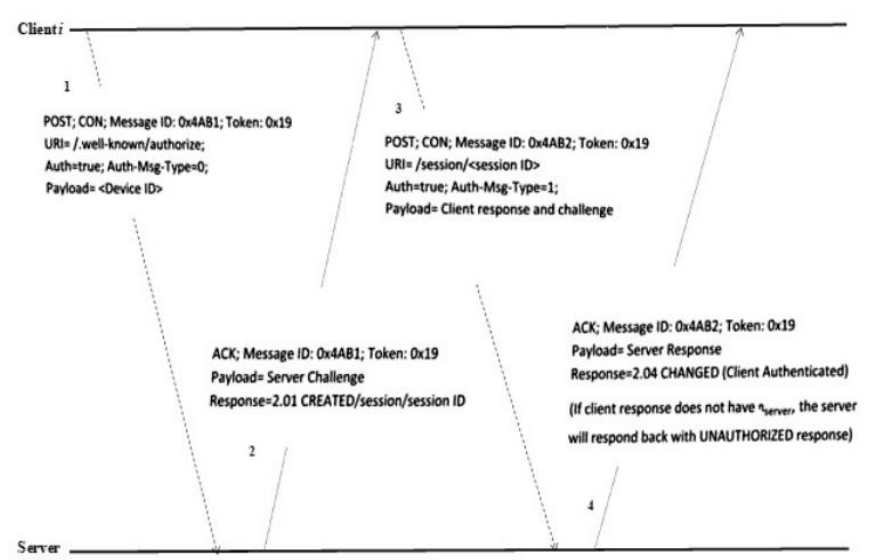

At long last, in information preprocessing, the information clearing is completed. The gathered information could be deficient that lacking credits esteems or certain ascribes of intrigue, loud that is an arbitrary mistake or change in a deliberate variable, and conflicting that containing errors in codes or names. In this examination, the incompletes (missing qualities) and commotion is mulled over.

\section{B. Data Stream Processing Layer}

Stream preparing layer is performed to deal with the information originating from various IoT sensors at ongoing after appropriate pre-handling. This handling depends on the idea of equal preparing in which different execution of cycles are performed simultaneously. Equal handling plan a program execute speedier on the grounds that there are numerous motors (CPUs) running it. The preparing of the information in proposed engineering begins with the information filtration. The filtration cycle is utilized to dispose of the futile information implies which isn't valuable for the handling and doesn't influence the aftereffects of the preparing. In the proposed engineering, the Kalman Filter (KF) is utilized to more accelerate the information preparing and separate the important and loud information. KF is used to complete the information filtration so as to channel the commotion from the information. It is a measurable technique and has a critical influence in detecting of this present reality information [42], [43]. When the filtration and extraction is done, the information is put away in a worker for load adjusting. Burden adjusting is conveyed as we perform equal handling. A heap balancer is utilized to disseminate the traffic over various equal processors so as to appropriate the heap. It expands the dependability of handling alongside the simultaneous clients limit.

\section{Decision-Making \& User-Interface Layer}

Subsequent to handling, results are conveyed to particular HAN for consistence and information is put away in data set for future pattern investigation.

In this stage, above all else the choice and prioritization is occurred. In choice and prioritization, the gadgets are chosen dependent on the boundaries gave by the purchasers.

The gadgets are chosen in two unique stages. In stage I, the HANs are organized dependent on their necessities and measures gave by them and in Phase II, the gadgets are chosen for a specific HAN. The information contains $s$ set of limited number of gadgets with partner cost and burden and yield having a lot of chosen gadgets. The I/O can be spoken to as follow: 


$$
\begin{gathered}
\text { Output: } \\
\mathrm{S}=\left\{\mathrm{s}_{1}, \mathrm{~s}_{2}, \mathrm{~s}_{3}\right\} \\
\mathrm{S} \subseteq \mathrm{D} \mid \operatorname{Min} \sum_{s \in S}^{n} C_{s} \\
\text { and } \sum_{s \in S}^{n} L_{s} \leq \text { MaxLoad }
\end{gathered}
$$

As we have to locate an ideal item/arrangement from limited set. This situation is remembered for combinatorial improvement. In such issues the conventional thorough looking isn't practical on the grounds that in such issues the ideal arrangements are discrete or will in general be discrete. Accordingly, we incline toward the dynamic programming approach and the backpack issue which is in the combinatorial enhancement. Dynamic Programming based 0/1 Knapsack Algorithm:: The one of the overall issue is the $0-1$ rucksack issue that restricts the number xi of imitations of all kind of section to 0 or 1 . For instance, different things set the rucksack to discover the maximum absolute worth. Each thing has specific weight and worth. Complete weight will consistently be not exactly fixed weight called W. So loads and estimations of things must be mulled over is appeared in Table I. Given a lot of $\mathrm{n}$ things from $1-\mathrm{n}$, all with a worth vi and a weight wi, with a limit of most extreme weight $\mathrm{W}$, can be numerically spoken to as:

$$
\operatorname{Max} \sum_{i=1}^{n} v_{i} w_{i}
$$

TABLE I: Knapsack Problem

\begin{tabular}{|l|l|l|}
\hline Item No. & Weight & Value \\
\hline 1 & 1 & 8 \\
\hline 2 & 3 & 6 \\
\hline 3 & 5 & 5 \\
\hline
\end{tabular}

subject to,

$$
\sum_{i=1}^{n} w_{i} x_{i} \leq W \text { and } x_{i} \in\{0,1\}
$$

where, $x_{i}$ denotes the instances of $i$ consist of the knapsack.

where, xi signifies the cases of I comprise of the backpack. When the ideal arrangements are acquired, at that point the conclusive outcomes are created and store in a specific stockpiling territory for occasion the executives. These outcomes can be utilized to play out the pattern examination and revealing.

\section{RESULTS AND ANALYSIS}

The security authentication module is implemented using NetDuino Plus 2 boards for the client and server interaction model. The experimental work is performed using .NET Micro Framework for resource-constrained devices with at least $256 \mathrm{~KB}$ of flash and $64 \mathrm{~KB}$ of RAM.

\section{A. Security Authentication using Proposed Architecture}

In this segment the trial results concerning security layer verification plot is given. In Figure 8, the correlation of the proposed verification plot with the CoAP-based DTLS usage (INDIGO) for cell phones is given. DTLS* represents the handshake between standard PC and an advanced cell where the PC works as a worker and the PDA goes about as a customer. On opposite, DTLS+ represents the PC as a customer and the advanced cell as a worker. Essentially, the CoAP messages are moved nonconcurrently over the UDP attachments in the proposed design. Every customer keeps record of the moved CON demands to look after track. At the point when a coordinating a RST reaction or is gotten for such messages, the transmission is viewed as effective. The normal reaction time for a solitary confirmable message for a payload of one byte is thought about against DTLS and the CoAP convention (with no security), as portrayed in Figure 9. Correspondingly, the normal memory utilization of a message at the accumulate time is likewise procured utilizing the Microsoft.SPOT.Native gathering. The proposed conspire is contrasted and existing ones for a confirmable message of 500 bytes appeared in Figure 10. An impressive amount of memory to the messages accumulate time) is dispensed by CoapBlip [35] and 
its variety TinyCoAP [36] which is a variation of the standard C libraries that need TinyOS component for its establishment on a sensor hub. Then again, HTTP/UDP has a low memory impression as it doesn't give an unwavering quality system or a solicitation/reaction coordinating.

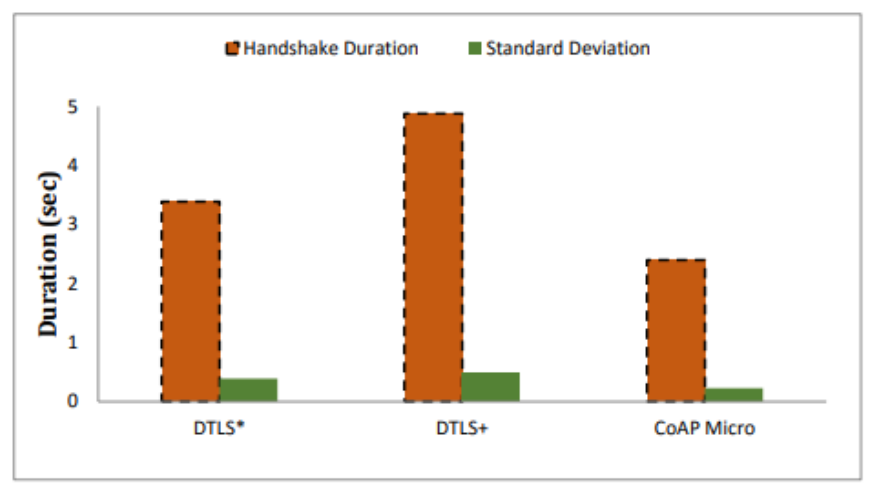

Fig. 8: Handshake Duration

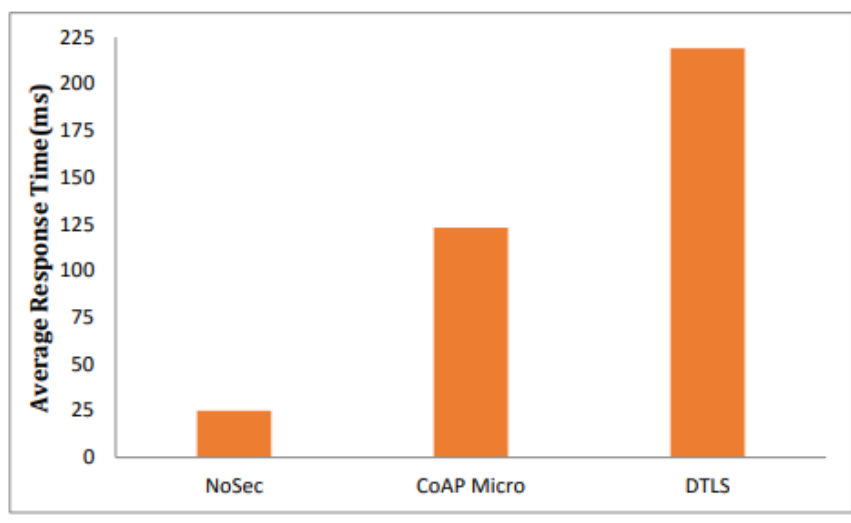

Fig. 9: Average Response Time

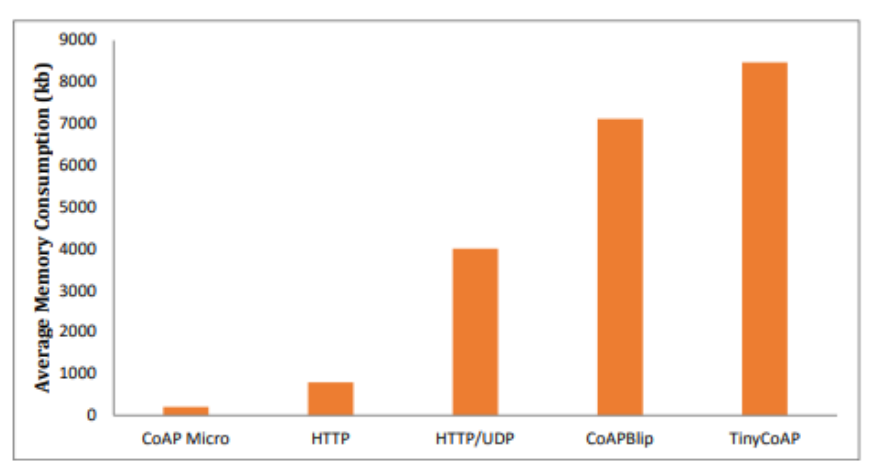

Fig. 10: Memory Consumption (500 Bytes payload)

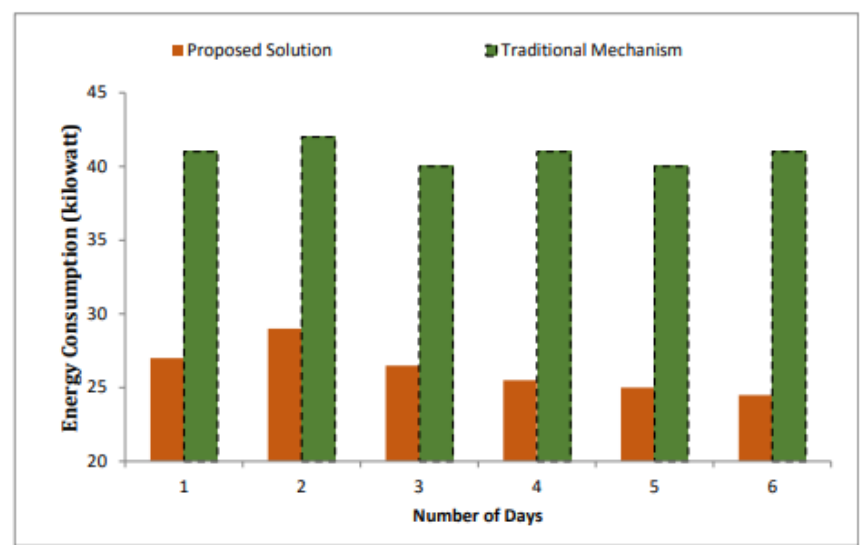

Fig. 11: Energy Consumption of Air-Condition

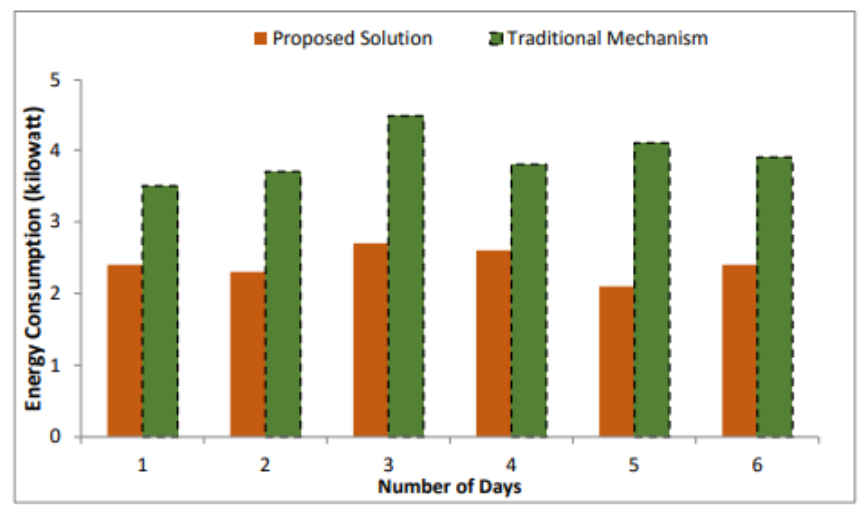

Fig. 12: Energy Consumption of Computer

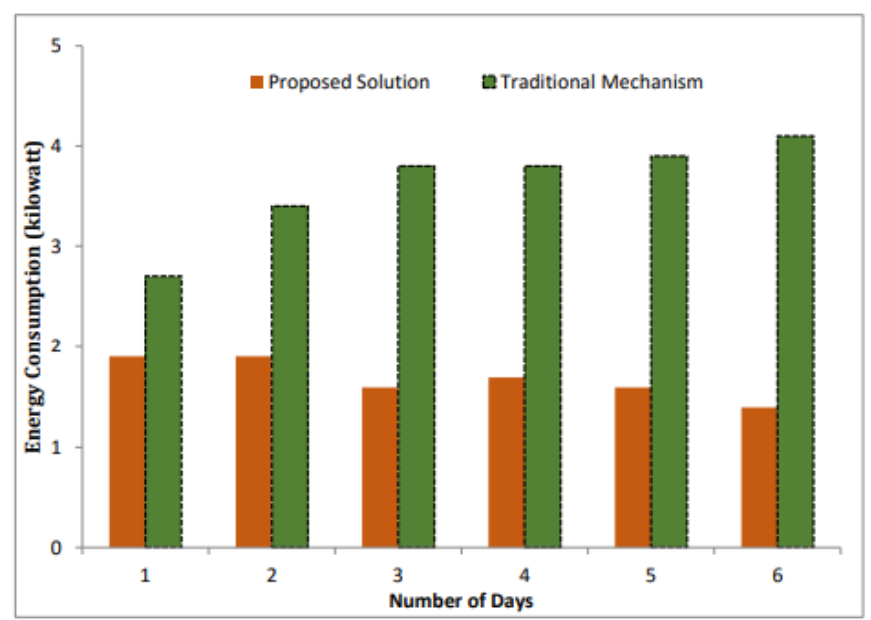

Fig. 13: Energy Consumption of Light Source 


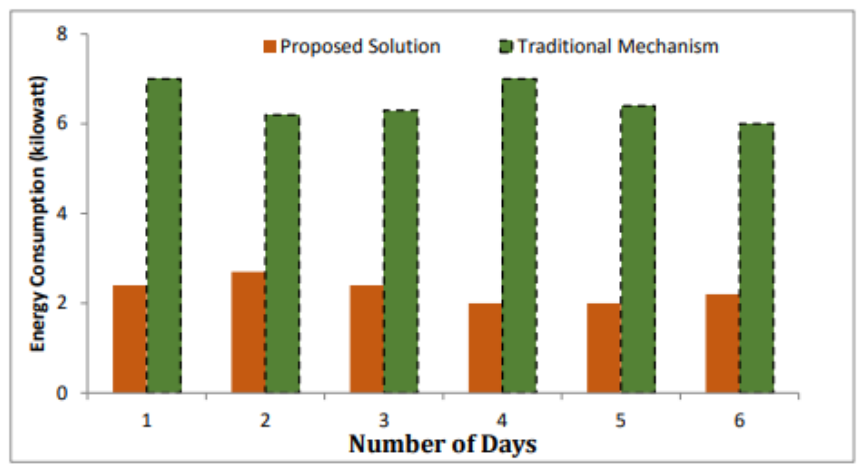

Fig. 14: Energy Consumption of Light Television

\section{Stream Processing and Decision-Making using Proposed Architecture}

In this segment the exploratory outcomes concerning stream handling utilizing huge Data investigation is given. The proposed DSM motor stream preparing is tentatively approved in a savvy home situation of various sensor. The vitality utilization of the sensors is tried by turning-on the gadgets arbitrarily. The said keen home is made out of one kitchen, four rooms, and two latrines. Each room has 4 gadgets and the kitchen is dispensed with 6 machines going from high to bring down vitality utilization. Essentially, the latrine has 2 apparatuses. In this manner, we have 26 sensors in the entire keen home situation. Besides, a portion of the gadgets that are lastingly present in the on-state, for example cooler, and so on are not thought about in the proposed conspire. The outcomes uncover that when the customers are consistently utilizing the gadgets the vitality utilization is essential increments. Then again, while applying the proposed design to the brilliant home condition the vitality usage significantly diminishes.

\section{CONCLUSION}

This paper proposes a safe and trusted multilayered DSM motor for a shrewd social society utilizing IIoT and Big Data examination. The proposed motor is an incorporated methodology so as to accomplish ideal DSM over an organization of Home Area Network
(HAN). Proposed design of concentrated Demand Side Management Engine takes Input from IoT sensors of HANs and performs validation and Big Data investigation for the determination and prioritization of gadgets of a specific HAN. The payload based verification conspire is used in proposed DSM motor to get the security. The payload-based encryption strategy uses a simple 4way handshake intend to confirm the taking an interest objects. Likewise, information stream into the brilliant social orders with regards to IIoT.

\section{REFERENCES}

[1] Gartner Incorporation. Gartner press release. http://www.gartner.com/newsroom/id/3598917 , Last accessed on April 7, 2018.

[2] Martin Strohbach, Holger Ziekow, Vangelis Gazis, and Navot Akiva. Towards a big data analytics framework for iot and smart city applications. In Modeling and processing for next-generation big-data technologies, Springer, 2015.

[3] Muhammad Babar and Fahim Arif. Real-time data processing scheme using big data analytics in internet of things based smart transportation environment. Journal of Ambient Intelligence and Humanized Computing, pages 1-11, 2018.

[4] Yunchuan Sun, Houbing Song, Antonio J Jara, and Rongfang Bie. Internet of things and big data analytics for smart and connected communities. IEEE Access, 4:766-773, 2016.

[5] M Mazhar Rathore, Awais Ahmad, Anand Paul, and Seungmin Rho. Urban planning and building smart cities based on the internet of things using big data analytics. Computer Networks, 101:63-80, 2016.

[6] IETF. Internet engineering task force. ttps://www.ietf.org, Last accessed on May 27, 2018. 
[7] Seung-Chul Son, Nak-Woo Kim, Byung-Tak Lee, Chae Ho Cho, and Jo Woon Chong. A time synchronization technique for coap-based home automation systems. IEEE Transactions on Consumer Electronics , 62(1):10-16, 2016.

[8] Marco Centenaro, Lorenzo Vangelista, Andrea Zanella, and Michele Zorzi. Long-range communications in unlicensed bands: The rising stars in the iot and smart city scenarios. IEEE Wireless Communications , 23(5):60-67, 2016.

[9] Shahid Raza, Ludwig Seitz, Denis Sitenkov, and Goran Selander. S3k: "Scalable security with symmetric keysdtls key establishment for theinternet of things. IEEE Transactions on Automation Science and Engineering, 13(3):1270-1280, 2016.

[10] Muhammad Babar and Fahim Arif. Smart urban planning using big data analytics to contend with the interoperability in internet of things. Future Generation Computer Systems, 77:6576, 2017.

[11] Muhammad Babar, Ataur Rahman, Fahim Arif, and Gwanggil Jeon. Energy-harvesting based on internet of things and big data analytics for smart health monitoring. Sustainable Computing: Informatics and Systems, 2017.

[12] Muhammad Babar and Fahim Arif. Smart urban planning using big data analytics based internet of things. In Proceedings of the 2017 ACM International Joint Conference on Pervasive and Ubiquitous Computing and Proceedings of the 2017 ACM International Symposium on Wearable Computers, pages 397-402. ACM, 2017.

\section{Cite this article as :}

Rupal Chaudhary, "Analysis and Development of Efficient DSM Engine for Smart Industrial Based on Big Data and IoT", International Journal of Scientific Research in Science and Technology (IJSRST), Online ISSN : 2395-602X, Print ISSN : 2395-6011, Volume 7 Issue 1, pp. 262-271, January-February 2020. Available at doi : https://doi.org/10.32628/IJSRST207534 Journal URL : http://ijsrst.com/IJSRST207534 